\title{
Up-to date of glomerular disease
}

\author{
Cristiana Rollino ${ }^{1}$ (i)
}

Received: 13 June 2016/Accepted: 20 June 2016/Published online: 2 July 2016

(C) Italian Society of Nephrology 2016

In spite of the wealth of literature published on glomerular diseases, clinicians still find themselves faced with numerous unsolved issues. Bearing this in mind, we have planned to provide an update on glomerular diseases in order to clarify the areas which still remain to be elucidated.

An obvious trend that nephrologists must come to terms with is the disappearance of the so-called 'idiopathic' diseases. The evolving classifications of glomerular diseases coupled with the identification of new forms of disease and of etiologies of well-known forms are slowly leading us along this path. Thirty-four podocytopathies have been identified to date [1]. Genetically defined, orphan podocytopathies may prove to be the next generation targets for precision medicine.

The glomerular classification recently published by the Mayo Clinic/Renal Pathology Society Consensus Report [2] has laid the cornerstone for the pathogenetic approach. Moving in the same direction is the subclassification of focal segmental glomerulosclerosis (FSGS). Different mechanisms of regulation of podocyte function may be altered in FSGF. These anomalies may all converge in a same histologic pattern which is the FSGS one. A hypothetical etiological/pathogenetic grouping may distinguish reactive podocytopathies, gene mutations, mechanical/ adaptive stress, deregulation of proliferation and local inflammation. Reactive forms of FSGS, for example, account for pauci-immune podocytopathies triggered by a circulating toxin/drug that is causative of severe injury in most podocytes [3].

Cristiana Rollino

cristiana.rollino@libero.it

1 S. Giovanni Bosco Hospital, University of Turin, Turin, Italy
The exact significance of C1q nephropathy, originally described as a form of FSGS, is still debated, and its relationship with outcome is variable. The histological spectrum could range from minimal change disease to mild mesangial proliferation to FSGS. Therefore the pathology of podocytopathies may have some overlapping features.

Immunoglobulin (Ig)M nephropathy is a highly controversial clinicopathological entity characterized by $\operatorname{IgM}$ diffuse deposits in the mesangium at immunofluorescence, whereas light microscopy identifies minimal glomerular lesions, hypercellularity and expansion of the mesangium or sclerotic focal segmental lesions. Recent experimental evidence suggests that IgM might have a pathogenic significance and not just be a marker of nonimmune injury; IgM may bind to endogenous neopitopes of glomerular cells and thus activate complement and amplify injury [4].

New light has also been shed on the glomerular association with infections. IgA-dominant infection-associated glomerulonephritis (GN) must be distinguished both from traditional IgA nephropathy and from acute post-infectious GN. These forms, which are characterized by the deposition of IgA in different sites and possible subepithelial C3 deposits with a hump-like aspect, should not be considered post-infectious GN [5] as the infection is often still present at the time of onset. This highlights the importance of therapeutic assessment.

From a clinical point of view, it is still debated whether corticosteroid (CS) treatment may be useful in the late stages of IgA nephropathy. Tesar et al., in a retrospective analysis based on the VALIGA study [6], compared 184 patients who received renin-angiotensin system blockers (RASB) with 184 patients who received RASB plus CS. The patients who received treatment with the addition of CS had a significantly slower decline of glomerular filtration rate than those who did not receive CS. These findings 
suggest that CS might also be useful in patients with advanced histological damage.

Another important but uncertain clinical acquisition is the role played by anti-phospholipase A2 receptors (APLA2r) in membranous GN (MGN)-this is a topic marked by light and shadow. APLA2r positivity in serum has been claimed to be highly suggestive of primary MGN, but current data suggest that it is still not specific enough to be able to avoid the screening for secondary forms of the disease. Hepatitis B virus-associated membranous nephropathy (HBV-MN) may be APLA2r-positive; an APLA2r-positive patient may be entering spontaneous remission; different anti-podocyte antibodies are identified, such as antibodies against anti-thrombospondin type-1 domain-containing 7A (THSD7A), present in about $10 \%$ of APLA2r-negative patients [7].

Finally, I would highlight the recognition of IgG4-related diseases (RD), in which the kidney can be involved either directly or indirectly. The most frequent direct renal manifestations of IgG4-RD are a tubulointerstitial nephritis and MGN [8].

While we await further advances in knowledge, such as on the possible practical role of proteomics in the diagnosis of renal disease, referred to by Mischak as "liquid biopsy" [9], it is important to assimilate and acquire with progressive confidence these recent significant evolutions in nephrology.

\section{Compliance with ethical standards}

Conflict of interest The author declares that he has no conflict of interest.
Ethical approval This article does not contain any studies with human participants performed by any of the authors.

\section{References}

1. Ranganathan S (2016) Causing nephrotic syndrome in children. Front Pediatr 4:1-12

2. Sethi S, Haas M, Markowitz GS et al (2016) Mayo clinic/renal pathology society consensus report on pathologic classification, diagnosis, and reporting of GN. JASN 27:1278-1287

3. Stokes MB, D’Agati VD (2014) Morphologic variants of focal segmental glomerulosclerosis and their significance. Adv Chronic Kidney Dis 21:400-407

4. Panzer SE, Laskowski J, Renner B et al (2015) IgM exacerbates glomerular disease progression in complement-induced glomerulopathy. Kidney Int 88:528-537

5. Glassock R, Alvarado A, Prosek J et al (2015) Staphylococcusrelated glomerulonephritis and poststreptococcal glomerulonephritis: why defining "post" is important in understanding and treating infection-related glomerulonephritis. Am $\mathrm{J}$ Kidney Dis 65:826-832

6. Tesar V, Troyanov S, Bellur S et al (2015) On behalf of the VALIGA study of ERA-EDTA Immunonephrology Working Group. Corticosteroids in IgA nephropathy: a retrospective analysis from the VALIGA study. JASN 26:2248-2258

7. Tomas NM, Beck LH, Meyer-Schwesinger C et al (2014) Thrombospondin type-1 domain-containing 7A in idiopathic membranous nephropathy. N Engl J Med 371:2277-2287

8. Saeki T, Kawano M (2014) IgG4-related kidney disease. Kidney Int 85:251-257

9. Mischak H (2015) Pro: urine proteomics as a liquid kidney biopsy: no more kidney punctures! Nephrol Dial Transplant 30:532-537 